\title{
End-point Parametrization and Guaranteed Stability for a Model Predictive Control Scheme
}

\author{
Siep Weiland* \\ Anton A. Stoorvogel ${ }^{\dagger} \ddagger$ \\ Andrei A. Tiagounov*
}

\begin{abstract}
In this paper we consider the closed-loop asymptotic stability of the model predictive control scheme which involves the minimization of a quadratic criterion with a varying weight on the end-point state. In particular, we investigate the stability properties of the (MPC-) controlled system as function of the end-point penalty and provide a useful parametrization of the class of end-point penalties for which stability of the controlled system can be guaranteed. The results are successfully applied for the implementation of an MPC controller of a binary distillation process.
\end{abstract}

\section{Introduction}

Model predictive or receding horizon controllers have received a great deal of attention and receive an ever growing interest for applications in industrial process control. Nevertheless, the stability of model predictive control schemes is difficult to analyze and few results are known which guarantee stability of model predictive controllers. See, for example, $[3,7-9,13,14,16]$ for various accounts on the stability of receding horizon controllers for linear and non-linear systems, with and without constrained inputs. It is well known that conditions on the terminal state are important to enforce stability of the controlled system. For linear time-invariant dynamical systems, this amounts to minimizing a quadratic cost criterion with an end-point penalty on the terminal state of the control horizon. In this paper we will analyze the stability properties of the controlled system as function of the end-point penalty of the criterion function. The effect of finite end-point penalties on the stability of receding horizon schemes has been investigated in $[1,12]$ for discrete time systems and in $[7,9,12]$ for continuous time systems. all these papers provide sufficient conditions for stability of controlled systems based on monotonicity results of Riccati equations and linear matrix inequalities. The main result in this paper provides an explicit parametrization of a class of end-point penalties for which exponential stability of the controlled system can be guaranteed. The result is based on a different technique and is a generalization of [15] to the case of non-square systems. We apply this result for

\footnotetext{
*Dept. of Elec. Eng., Eindhoven University of Technology, P.O. Box 513, 5600 MB Eindhoven, E-mail: \{s.weiland, a.tiagounov\}@ tue.nl

$\dagger$ Dept of Math. and Comp. Science, Eindhoven University of Technology, P.O. Box 513, 5600 MB Eindhoven, E-mail: a.a.stoorvogel@tue.nl

¥Dept. of Information Technology and Systems, Delft University of Technology, P.O. Box 5031, 2600 GA Delft, The Netherlands
}

the synthesis of a model predictive controller for a binary distillation column.

The problem is formalized in Section 2, main results are collected in Section 3, the application is given in Section 4. All proofs are deferred to the Appendix.

As for the notation, $\mathscr{L}_{2}$ denotes the standard Hilbert space of complex valued functions which are square integrable on the unit circle. We denote by $\ell_{2}$ the normed space of discrete time series (on $\mathbb{Z}$ ) which are square summable. $R H_{\infty}$ is the Hardy space of complex valued rational functions, which are bounded on $\{z \in \mathbb{C}|| z \mid \geq 1\}$.

\section{Problem formulation}

Consider a linear discrete-time plant described by

$$
y=P u
$$

where $u$ is the $m$ dimensional input, $y$ is the $p$ dimensional output, and $P(z)$ is a real rational proper transfer function. Identify the plant with its graph

$$
\operatorname{graph}(P):=\left\{\operatorname{col}(u, y) \in \mathcal{L}_{2} \mid y=P u\right\}
$$

where $\operatorname{col}(\cdot, \cdot)$ means stacking subsequent entries. Hence, $\operatorname{graph}(P)$ is the collection of all possible $\mathcal{L}_{2}$-bounded inputoutput pairs which are compatible with the plant. It is well known [17] that $P$ allows a right coprime factorization* $P=N D^{-1}$ over $R H_{\infty}$ and that for any such factorization

$$
\operatorname{graph} P=\operatorname{im}\left(\begin{array}{l}
N \\
D
\end{array}\right)
$$

where $\operatorname{col}(N, D)$ is viewed as a multiplicative operator with domain $\mathcal{L}_{2}^{m}$. Such a factorization is normalized if $G:=$ $\operatorname{col}(N, D)$ is inner, that is, $G \in R H_{\infty}^{(m+p) \times m}$ and $G$ is norm preserving in the sense that $\|G v\|_{2}=\|v\|_{2}$ for all $v \in \mathcal{L}_{2}$. Normalized right coprime factorizations of $P$ exist [17] and we suppose that

$$
\begin{cases}x(k+1) & =A x(k)+B v(k) \\ w(k) & =C x(k)+D v(k)\end{cases}
$$

*That is, $N, D \in R H_{\infty}, D$ is non-singular as a rational matrix, and the pair $(N, D)$ is right coprime in the sense that there exist $X, Y \in R H_{\infty}$ such that $X N+Y D=I$. 
is a minimal state representation of $G=\operatorname{col}(N, D)$ with $N, D$ a normalized right coprime factorization of $P$. Here, $w=\operatorname{col}(u, y)$ is called the external variable, $v$ the driving variable, $x$ is the state and $G(z):=C(I z-A)^{-1} B+D$ is inner. Under the given conditions, (1) is said to be an isometric state representation of the plant. There exist efficient algorithms to convert the transfer function $P$ to an isometric state representations (1) and it is a standard result [4] that (1) is an isometric state representation if and only if there exists $Q=Q^{\top}>0$ such that

$$
\left(\begin{array}{ll}
A & B \\
C & D
\end{array}\right)^{\top}\left(\begin{array}{ll}
Q & 0 \\
0 & I
\end{array}\right)\left(\begin{array}{ll}
A & B \\
C & D
\end{array}\right)=\left(\begin{array}{ll}
Q & 0 \\
0 & I
\end{array}\right)
$$

In fact, $Q>0$ is uniquely defined by (2) and is called the observability gramian of (1). The controllability gramian associated with (1) is the unique positive solution $P$ of

$$
A P A^{\top}+B B^{\top}=P
$$

which in a suitable (balanced) basis of the state space will be an identity matrix. In that case we will call (1) a canonical isometric state representation (CISR) of the plant.

Suppose that the plant is represented by a canonical isometric state representation (1). Let $t \in \mathbb{Z}_{+}$be the current time, $T_{t}:=[t, t+N-1]$ a control horizon of length $0<N<\infty$ and consider the quadratic objective function

$$
J_{t}\left(x_{t}, v\right):=\sum_{k \in T_{t}}\|w(k)\|^{2}+\|x(t+N)\|_{E}^{2}
$$

where $w(k)=\operatorname{col}(u(k), y(k))$ satisfies (1) with $k \in T_{t}$, $x_{t}:=x(t)$ as initial state and $v: T_{t} \rightarrow \mathbb{R}^{m}$ the driving variable. Here, $\|x\|^{2}=\langle x, x\rangle$ and $\|x\|_{E}^{2}:=\langle x, E x\rangle$ with $E=E^{\top}$. The criterion (3) therefore involves a weighted end-point penalty on the state which is reached at the endpoint of the optimization interval $T_{t}$. We will not assume that $E \geq 0$ and hence the end-point penalty may, at least in principle, be indefinite. Note that the summation in (3) ranges over a time dependent interval of fixed length $N$. Whenever finite, the optimal cost is defined as

$$
J_{t}^{*}\left(x_{t}\right):=\inf _{v: T_{t} \rightarrow \mathbb{R}^{m}} J_{t}\left(x_{t}, v\right) .
$$

The (open-loop) optimization problem at time instant $t$ amounts to minimizing (3) subject to the equations (1). Whenever it exists, $v_{t}^{*}: T_{t} \rightarrow \mathbb{R}^{m}$ denotes the (or a) minimizing control for (3) in that

$$
J_{t}^{*}\left(x_{t}\right):=J_{t}\left(x_{t}, v_{t}^{*}\right)
$$

In a receding horizon setting only the first time instant $v_{t}^{*}(t)$ of $v_{t}^{*}$ is implemented as driving variable input for the system (1). This means that at time $t, v(t)=v_{t}^{*}(t)$ is fed in (1), and the next state $x_{t+1}=x(t+1)=A x_{t}+B v_{t}^{*}(t)$ is taken as initial state for a renewed minimization of $J_{t+1}\left(x_{t+1}, \cdot\right)$ at the next sample time $t+1$. Repeating this calculation for all $t \in \mathbb{Z}_{+}$results in the sequence

$$
v_{\mathrm{mpc}}:=\left\{v_{t}^{*}(t)\right\}_{t \in \mathbb{Z}_{+}}
$$

which we will call the receding horizon or model predictive controller for the plant.

The problem we address in this paper concerns the stability of the system (1) when controlled by (5).

Definition 2.1 (Problem formulation) The receding horizon control problem with stability [RHCPS] is to find $E=$ $E^{\top}$ such that the controlled system (1)-(5) is exponentially stable in the sense that there exist constants $c_{1}>0$ and $0<c_{2}<1$ such that $\|x(k)\| \leq c_{1}\left\|x_{0}\right\| c_{2}^{k}$ for all $k \in \mathbb{Z}_{+}$ and for all $x_{0} \in \mathbb{R}^{n}$. In that case the sequence (5) is called stabilizing for the plant.

This notion of stability is also called global exponential stability, cf. [18].

Remark 2.2 $\operatorname{graph}(P)$ is well defined for both stable and unstable systems. If $P$ has no poles on the unit circle, the transfer function $P$ defines a mapping $P: \mathscr{L}_{2} \rightarrow \mathcal{L}_{2}$ so that $\mathcal{L}_{2}$ can be taken as domain of $P$ and $\operatorname{graph}(P)=\mathscr{L}_{2} \times$ $P \mathscr{L}_{2}$. This means that the input $u$ is a free variable in $\mathscr{L}_{2}$. If $P$ does have poles on the unit circle, $\operatorname{graph}(P)$ is still well defined, but the input is no longer a free variable in $\mathcal{L}_{2}$.

Remark 2.3 For practical reasons it may be desirable to incorporate different weights of the components of $w$ in the criterion function (3). This means that $\|w(k)\|^{2}$ in (3) needs to be replaced by the quadratic form $\|w(k)\|_{W}^{2}$ where $W=W^{\top} \geq 0$ is a non-negative definite weighting matrix. Redefining $w$ in (1) by $w^{\prime}=W^{1 / 2} w$ implies that we may assume, without loss of generality, that these weightings have been incorporated already.

Remark 2.4 If the input $u$ is viewed as control variable, then the MPC control law (5) defines the control input $u_{\mathrm{mpc}}:=\left(\begin{array}{ll}I_{m} & 0\end{array}\right) G v_{\mathrm{mpc}}$ for the plant $P$ in an obvious way.

\section{Main results}

Consider the recursions

$$
\begin{aligned}
\Pi_{k}= & A^{\top} \Pi_{k+1} A+C^{\top} C-\left(A^{\top} \Pi_{k+1} B+C^{\top} D\right) \times \\
& \left(B^{\top} \Pi_{k+1} B+D^{\top} D\right)^{-1}\left(B^{\top} \Pi_{k+1} A+D^{\top} C\right) \\
F_{k}= & \left(B^{\top} \Pi_{k+1} B+D^{\top} D\right)^{-1}\left(B^{\top} \Pi_{k+1} A+D^{\top} C\right) .
\end{aligned}
$$

with $\Pi_{N}=E$. Assuming that the inverses exist, this defines the matrix sequences $\left\{\Pi_{k}\right\}_{k=0}^{N}$ and $\left\{F_{k}\right\}_{k=0}^{N-1}$. The facts are that

$$
J_{t}^{*}>-\infty
$$

if and only if the matrices $B^{\top} \Pi_{k+1} B+D^{\top} D \geq 0$ for all $k=0, \ldots, N-1$. The minimizing control $v_{t}^{*}$ satisfying (4) exists and is unique if and only if $B^{\top} \Pi_{k+1} B+D^{\top} D>0$ for 
all $k=0, \ldots, N-1$. In that case, the minimizing control in closed-loop form is

$$
v_{t}^{*}(k):=F_{k-t} x(k), \quad k \in T_{t}
$$

with $x(k)$ the solution of (1) with $x(t)=x_{t}$ and $v(k)=$ $v_{t}^{*}(k)$, whereas in open-loop form it is

$$
v_{t}^{*}(k):=F_{k-t} \Phi(k, t) x_{t}, \quad k \in T_{t}
$$

with $\Phi$ being the transition matrix for $x(k+1)=(A+$ $\left.B F_{k-t}\right) x(k)$. In either case, $J^{*}\left(x_{t}\right)=x_{t}^{\top} \Pi_{0} x_{t}^{\top}$ is the optimal cost. We will assume that such a minimizing control exists. By (5),

$$
v_{\mathrm{mpc}}(t)=F_{0} x(t), \quad t \in \mathbb{Z}_{+}
$$

is then a state feedback implementation of the receding horizon control law. Since $\left\{\Pi_{k}\right\}_{k=0}^{N}$ and $\left\{F_{k}\right\}_{k=0}^{N-1}$ do not depend on the time $t$, these sequences can be calculated off-line and the MPC control law (8) is time invariant. The receding horizon control problem with stability is therefore solved if and only if the eigenvalues of

$$
A_{\mathrm{cl}}:=A+B F_{0}
$$

belong to the open unit disc of complex points $z \in \mathbb{C}$ with $|z|<1$.

It is well known that the control law (5) will, in general, not stabilize the system (1) if the end-point state is not weighted in the optimization criterion, i.e., if $E=0$. It is for this reason that exponential stability is sometimes enforced by minimizing a quadratic criterion over $T_{t}$ subject to the endpoint constraint $x(t+N)=0$ (deadbeat control). In view of the criterion function (5), this constraint has the interpretation of an infinite weight on the state $x(t+N)$. See, for example, $[6,8,11]$. This requirement is certainly undesirable from a practical point of view, especially when the control horizon $N$ is small. In [13] it has been shown that stability is achieved for $E=Q$ where $Q$ is the observability gramian of (1). If $E=\Pi$ with $\Pi$ the unique non-negative definite steady-state solution (i.e. $\Pi_{k}=\Pi \geq 0$ for all $0 \leq k \leq N$ ) of (6), then $F_{k}$ is independent of $k$ and $N$, and the receding horizon controller (8) will stabilize the system independent of the length $N$ of the control horizon. Hence, the receding horizon controller is stabilizing if $E=\Pi$. See also [9] for stability conditions based on linear matrix inequalities.

There exists an obvious intuitive idea that an increase of the end-point penalty will preserve exponential stability. We were not able to establish this result. Let us formally present our conjecture:

Conjecture 3.1 Suppose that the receding horizon controller (5) is stabilizing with $E=E^{\top}$, then it is also stabilizing for all $E^{\prime} \geq E$.

The following theorem is the main contribution of this paper.
Theorem 3.2 Suppose that (1) is a canonical isometric state representation of the plant. Let $N>0$ denote the control horizon and let $Q$ be the observability gramian defined in (2). Then

1. If $E-Q$ and $A$ are both non-singular, then

$$
A_{c l}=\left(X_{1}-I\right)\left(X_{1}-A A^{\top}\right)^{-1} A \text {. }
$$

where $X_{1}=X_{1}^{\top}$ is matrix which is uniquely defined by the recursion

$$
\left\{\begin{array}{l}
A X_{k} A^{\top}=X_{k+1} \\
X_{N}=(E-Q)^{-1}+I
\end{array} .\right.
$$

Moreover, when $Q-I \leq E<Q$ then $X_{1} \geq 0$. In particular, (5) stabilizes $G$ if (10) has its eigenvalues in $|z|<1$.

\section{For all E satisfying:}

$$
E=Q+\left(A^{N-1} X\left(A^{\top}\right)^{N-1}-I\right)^{-1}
$$

where $X=X^{\top}$ commutes with $A A^{\top}$ and satisfies $X^{2}>A A^{\top}$, we have that the receding horizon controller (5) achieves exponential stability of the controlled system.

We would like to emphasize the importance of the above result. First, Theorem 3.2 gives an explicit set of end-point penalties $E$ for which the receding horizon optimal controller stabilizes the system. Since $Q-I \leq 0$, this set may include non-positive definite end-point weights. Second, note that the conditions for $X$ are independent of the control horizon $N$, the limit

$$
\lim _{N \rightarrow \infty}\left(A^{N} X\left(A^{\top}\right)^{N}-I\right)^{-1}=-I
$$

and the right-hand side of (12) therefore converges to the negative semi-definite matrix $Q-I$ if the control horizon $N$ tends to infinity. This means that the receding horizon control law is stabilizing with non-positive end-point penalties, asymptotically as $N \rightarrow \infty$. Third, for any fixed $X$ commuting with $A A^{\top}$ and satisfying $X^{2}>A A^{\top}$, the righthand side of (12) is a non-increasing function of $N$. This implies that the receding horizon controller stabilizes the system with decreasing end-point penalties, as the control horizon $N$ increases. Note that finding suitable $X$ commuting with $A A^{\top}$ is trivial. For instance $X=\alpha I$ commutes with $A A^{\top}$ and satisfies $X^{2}>A A^{\top}$ for $\alpha>1$. It is an interesting consequence of the analysis that the recursion (11) is relevant for the derivation. As shown in the Appendix, $X_{k}$ is related to $\Pi_{k}$ according to $X_{k}=\left(\Pi_{k}-Q\right)^{-1}+I$ for all $k \leq N$. 


\section{MPC of a binary distillation column}

\section{1 the plant}

As an application of the theory of the preceding sections we consider a case study of an MPC controller for a binary distillation column ( [5]) as depicted in Figure 1. The distillation column consists of $n$ stages, including reboiler and condenser which are numbered from top to bottom. The feed enters the column at stage $n_{\mathrm{f}}$ with feed flow $F[\mathrm{kmol} / \mathrm{hr}] . L$ $[\mathrm{kmole} / \mathrm{hr}]$ denotes the reflux flow rate of the condenser, $V_{\mathrm{b}}$ [kmole $/ \mathrm{hr}]$ is the boilup flow rate and $u=\operatorname{col}\left(L, V_{\mathrm{b}}, F\right)$ is taken as the input variable of the plant. The output of the plant is given by $y=\operatorname{col}\left(X_{\mathrm{d}}, X_{\mathrm{b}}\right)$ where $X_{\mathrm{d}}$ and $X_{\mathrm{b}}$ [mole fraction] denote the distillate and bottom compositions, respectively.

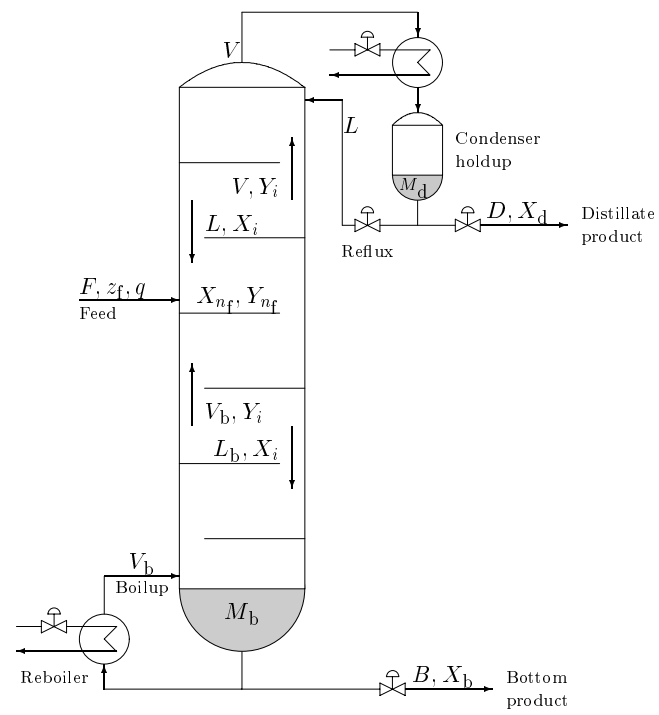

Figure 1: Binary distillation column.

\section{2 the model}

The stages above the feed (index $i<n_{\mathrm{f}}$ ) define the enriching section and those below the feed (index $i>n_{\mathrm{f}}$ ) the stripping section of the column. The material balance equations for the feed stage and the stages in the stripping section of the column are affected by the continuous feed to the column and the withdrawal of the bottom product from the reboiler. In particular, we have that the liquid flow rate in the stripping section is defined as $L_{\mathrm{b}}=L+q F$ where $q$ is a constant. The vapor flow rate in the enriching section is given by $V=V_{\mathrm{b}}+(1-q) F$. The distillate and bottom product flow rates are $D=V-L$ and $B=L_{\mathrm{b}}-V_{\mathrm{b}}$, respectively. Denote by $X_{i}$ and $Y_{i}$ [mole fraction] the liquid and vapor compositions of stage $i$, respectively. For constant liquid holdup conditions, the material balance for the column are given as follows.

$$
\begin{aligned}
M_{\mathrm{d}} \frac{d X_{1}}{d t} & =V Y_{2}-(L+D) X_{1} \\
M \frac{d X_{i}}{d t} & =L\left(X_{i-1}-X_{i}\right)+V\left(Y_{i+1}-Y_{i}\right), 1<i<n_{\mathrm{f}}
\end{aligned}
$$

$$
\begin{aligned}
M \frac{d X_{\mathrm{f}}}{d t} & =L X_{\mathrm{f}-1}-L_{\mathrm{b}} X_{\mathrm{f}}+V_{\mathrm{b}} Y_{\mathrm{f}+1}-V Y_{\mathrm{f}}+F z_{\mathrm{f}} \\
M \frac{d X_{i}}{d t} & =L_{\mathrm{b}}\left(X_{i-1}-X_{i}\right)+V_{\mathrm{b}}\left(Y_{i+1}-Y_{i}\right), n_{\mathrm{f}}<i<n \\
M_{\mathrm{b}} \frac{d X_{n}}{d t} & =L_{\mathrm{b}} X_{n-1}-V_{\mathrm{b}} Y_{n}-B X_{n} .
\end{aligned}
$$

The nonlinear vapor-liquid equilibrium equation describes the relation between compositions on each stage:

$$
Y_{i}=\frac{\alpha X_{i}}{1+(\alpha-1) X_{i}}, \quad i=1, \ldots, n .
$$

This yields a nonlinear model $\dot{x}=f(x, u), y=g(x, u)$ of state dimension $n$. We linearize the model around a linearization point $\operatorname{col}(u, x, y)=\operatorname{col}\left(u^{*}, x^{*}, y^{*}\right)$ representing a vector of steady-state operation of the nonlinear system. This yields a continuous time linear model with transfer function $P(s)=C(s I-A)^{-1} B+D$, where $A=\frac{\partial f}{\partial x}\left(x^{*}, u^{*}\right), B=\frac{\partial f}{\partial u}\left(x^{*}, u^{*}\right) C=\frac{\partial g}{\partial x}\left(x^{*}, u^{*}\right)$ and $D=\frac{\partial g}{\partial u}\left(x^{*}, u^{*}\right)$. Here, the Vapor-Liquid Equilibrium equation is linearized around the operating points

$$
Y_{i}^{*}=\frac{\alpha}{\left(1+(\alpha-1) X_{i}^{*}\right)^{2}}, \quad i=1, \ldots, N .
$$

The matrix $A$ is non-zero for the entries

$$
\begin{aligned}
A_{i, i}= \begin{cases}-\frac{(L+D)}{M_{\mathrm{d}}} & i=1, \\
-\frac{\left(L+V Y_{i}^{*}\right)}{M} & i=2, \ldots, n_{\mathrm{f}}-1, \\
-\frac{\left(L_{\mathrm{b}}+V Y_{i}^{*}\right)}{M} & i=n_{\mathrm{f}}, \\
-\frac{\left(L_{\mathrm{b}}+V_{\mathrm{b}} Y_{i}^{*}\right)}{M} & i=n_{\mathrm{f}}+1, \ldots, n-1, \\
-\frac{\left(B+V_{\mathrm{b}} Y_{i}^{*}\right)}{M_{\mathrm{b}}} & i=n .\end{cases} \\
A_{i, i-1}= \begin{cases}\frac{L}{M} \quad i=2, \ldots, n_{\mathrm{f}}, \\
\frac{L_{\mathrm{b}}}{M} \quad i=n_{\mathrm{f}}+1, \ldots, n-1, \\
\frac{L_{\mathrm{b}}}{M_{\mathrm{b}}} \quad i=n .\end{cases} \\
A_{i, i+1}= \begin{cases}\frac{V Y_{i+1}^{*}}{M_{\mathrm{d}}} & i=1, \\
\frac{V Y_{i+1}^{*}}{M} & i=2, \ldots, n_{\mathrm{f}}-1, \\
\frac{V_{\mathrm{b}} Y_{i+1}^{*}}{M} & i=n_{\mathrm{f}}, \ldots, n-1,\end{cases}
\end{aligned}
$$

The $i$ th row $B_{i}$ of $B$ is given by

$$
\left\{\begin{array}{llll}
(0 & Y_{i+1}-X_{i}^{*} & \left.(1-q)\left(Y_{i+1}-X_{i}^{*}\right)\right) \frac{1}{M_{\mathrm{d}}} & i=1, \\
\left(X_{i-1}^{*}-X_{i}^{*}\right. & Y_{i+1}-Y_{i} & \left.(1-q)\left(Y_{i+1}-Y_{i}\right)\right) \frac{1}{M} & 1<i<n_{\mathrm{f}}, \\
\left(X_{i-1}^{*}-X_{i}^{*}\right. & Y_{i+1}-Y_{i} & \left.-q X_{i}^{*}-(1-q) Y_{i}+z_{\mathrm{f}}\right) \frac{1}{M} & i=n_{\mathrm{f}}, \\
\left(X_{i-1}^{*}-X_{i}^{*}\right. & Y_{i+1}-Y_{i} & \left.q\left(X_{i-1}^{*}-X_{i}^{*}\right)\right) \frac{1}{M} & n_{\mathrm{f}}<i<n, \\
\left(X_{i-1}^{*}-X_{i}^{*}\right. & -Y_{i}+X_{i}^{*} & \left.q\left(X_{i-1}^{*}-X_{i}^{*}\right)\right) \frac{1}{M_{\mathrm{b}}} & i=n .
\end{array}\right.
$$

Finally,

$$
C=\left(\begin{array}{lllll}
1 & 0 & \cdots & 0 & 0 \\
0 & 0 & \cdots & 0 & 1
\end{array}\right)
$$

and $D=0$.

\subsection{MPC control}

Physical parameters of the plant are obtained from [5] and given in Table 1. Using a bilinear transformation, the con- 


\begin{tabular}{lll}
\hline$n$ & Number of stages & 20 \\
$n_{\mathrm{f}}$ & Feed stage & 6 \\
$M \mathrm{~d}$ & Condenser holdup & $200[\mathrm{kmol}]$ \\
$M \mathrm{~b}$ & Reboiler holdup & $400[\mathrm{kmol}]$ \\
$M$ & Stage holdup & $50[\mathrm{kmol}]$ \\
$x_{\mathrm{f}}$ & Feed composition & $0.5[\mathrm{~mole}$ fraction $]$ \\
$q$ & Feed liquid fraction & 1 \\
$\alpha$ & Relative volatility & 2.46 \\
$L^{*}$ & Reflux flow & $1090[\mathrm{kmol} / \mathrm{hr}]$ \\
$V_{\mathrm{b}}^{*}$ & Boilup vapor flow & $1575[\mathrm{kmol} / \mathrm{hr}]$ \\
$F^{*}$ & Feed flow & $1000[\mathrm{kmol} / \mathrm{hr}]$ \\
\hline
\end{tabular}

Table 1: Operating point data

tinuous time linearized plant has been discretized with sample time $T=0.08$ [hrs] to obtain a transfer function $P(z)$. Following the suggestion of Remark 2.3 we incorporate suitable weightings in the optimization criterion and define

$$
w=W^{1 / 2} \operatorname{col}(u, y), \quad W=\operatorname{diag}\left(W_{u}, W_{y}\right)
$$

with $W_{u}=10^{-6} * I$ and $W_{y}=I$. The graph $W^{1 / 2} \operatorname{graph}(P)$ is represented by an isometric state model of the form (1) with $n=20, m=3$ and $p=2$. The MPC controller (5) is implemented with various end-point penalties $E$ and control horizons $N$. Results are given in Table 2 where $c_{2}$, defined in Definition 2.1 is the smallest exponential decay of the controlled system. The results show that the reced-

\begin{tabular}{lll}
\hline$E=Q$ & $N=100$ & $c_{2}=0.8974$ \\
$E=\Pi$ & $N=100$ & $c_{2}=0.8974$ \\
$E=0$ & $N=10$ & $c_{2}=0.9244$ \\
$E=10^{4} \times Q$ & $N=10$ & $c_{2}=0.8262$ \\
\hline
\end{tabular}

Table 2: Operating point data

ing horizon controller provides exponential stability of the controlled system in all cases.

\section{APPENDIX: proof of main result}

\section{Proof of Theorem 3.2}

1. To prove the first item, suppose that $E-Q$ and $A$ are nonsingular. Use (2) to eliminate $C$ and $D$ from the recursions (6) and (7). With $\Psi_{k}:=\Pi_{k}-Q$ this yields, after some straightforward manipulation,

$$
\left(\begin{array}{c}
\Psi_{k} \\
F_{k}
\end{array}\right)=\left(\begin{array}{c}
A^{\top} \\
-B^{\top}
\end{array}\right) \Psi_{k+1}\left(B B^{\top} \Psi_{k+1}+I\right)^{-1} A .
$$

Hence,

$$
\begin{aligned}
A+B F_{k} & =\left[I-B B^{\top} \Psi_{k+1}\left(B B^{\top} \Psi_{k+1}+I\right)^{-1}\right] A \\
& =\left(B B^{\top} \Psi_{k+1}+I\right)^{-1} A .
\end{aligned}
$$

Since (1) is a canonical isometric state representation, we can use the identity $B B^{\top}=I-A A^{\top}$ to eliminate $B B^{\top}$ from the $\Psi_{k}$ recursion. We now claim that that all elements of $\left\{\Psi_{k}\right\}_{k=0}^{N}$ are invertible. Indeed, $\Psi_{N}=\Pi_{N}-Q=E-Q<$ 0 is invertible, and with the induction hypothesis that $\Psi_{k+1}$ is invertible for $0<k<N$, we have that

$$
\begin{aligned}
\Psi_{k} & =A^{\top} \Psi_{k+1}\left(\left[I-A A^{\top}\right] \Psi_{k+1}+I\right)^{-1} A \\
& =A^{\top}\left(I-A A^{\top}+\Psi_{k+1}^{-1}\right)^{-1} A \\
& =\left(A^{-1}\left[\Psi_{k+1}^{-1}+I\right]\left(A^{\top}\right)^{-1}-I\right)^{-1}
\end{aligned}
$$

i.e., $\Psi_{k}$ is invertible. Hence, $\Psi_{k}$ is invertible for all $0 \leq k \leq$ $N$. This yields

$$
\begin{aligned}
\Psi_{k} & =A^{\top} \Psi_{k+1}\left(\left[I-A A^{\top}\right] \Psi_{k+1}+I\right)^{-1} A \\
& =A^{\top}\left(I-A A^{\top}+\Psi_{k+1}^{-1}\right)^{-1} A \\
& =\left(A^{-1} A^{-\top}-I+A^{-1} \Psi_{k+1}^{-1} A^{-\top}\right)^{-1} .
\end{aligned}
$$

Taking the inverse on both sides yields, after some rearrangements,

$$
A\left(\Psi_{k}^{-1}+I\right) A^{\top}=\Psi_{k+1}^{-1}+I
$$

Now set $X_{k}=\Psi_{k}^{-1}+I, X_{N}=(E-Q)^{-1}+I$ to infer (11). It follows that

$$
\begin{aligned}
A+B F_{k} & =\left(B B^{\top} \Psi_{k+1}+I\right)^{-1} A \\
& =\left(\left(I-A A^{\top}\right) \Psi_{k+1}+I\right)^{-1} A \\
& =\left(\left(\Psi_{k+1}+I\right)-A A^{\top} \Psi_{k+1}\right)^{-1} A \\
& \left.=\left(\left(\Psi_{k+1}^{-1}+I\right)-I\right)\left(\Psi_{k+1}^{-1}+I\right)-A A^{\top}\right)^{-1} A \\
& =\left(X_{k+1}-I\right)\left(X_{k+1}-A A^{\top}\right)^{-1} A
\end{aligned}
$$

which yields $A_{\mathrm{cl}}$ by setting $k=0$. This proves (10). We next show that the sequence $\left\{X_{k}\right\}_{k=0}^{N}$ consists of nonpositive elements whenever $Q-I \leq X \leq Q$. To see this, first observe that for input-balanced isometric state representations $Q \leq I$. Consequently,

$$
\begin{aligned}
& Q-I \leq E<Q \Longleftrightarrow 0<Q-E \leq I \Longleftrightarrow \\
& (Q-E)^{-1} \geq I \Longleftrightarrow X_{N}=(E-Q)^{-1}+I \leq 0 .
\end{aligned}
$$

But if $X_{N} \leq 0$, then (11) guarantees that $X_{k} \leq 0$ for all $0 \leq k \leq N$.

2. The proof of item 2 is quite straightforward when we use the property of commuting $X$ and $A A^{\top}$. Note that, when $A$ is invertible we have by construction, $X_{1}=X$ and hence:

$$
A_{\mathrm{cl}}=(X-I)\left(X-A A^{\top}\right)^{-1} A .
$$

In general, we can establish by a perturbation argument that the expression (14) still holds when $A$ is singular. Define $Y=A A^{\top}$. From our expression for $A_{\mathrm{cl}}$ it is clear that $A_{\mathrm{cl}}$ is asymptotically stable whenever

$$
(X-I)(X-Y)^{-1} Y(X-Y)^{-1}(X-I)<I
$$


Since all matrices in this expression commute we can simplify this expression. Clearly we equivalently need to establish:

$$
(X-Y)^{-1}(X-I)^{2} Y(X-Y)^{-1}<I
$$

But we have:

$$
\begin{aligned}
X^{2}>Y & \\
(I-Y)^{1 / 2} X^{2}(I-Y)^{1 / 2} & >(I-Y)^{1 / 2} Y(I-Y)^{1 / 2} \\
X^{2}(I-Y) & >Y(I-Y) \\
X^{2}(Y-I) & <Y(Y-I) \\
X^{2} Y+Y & <X^{2}+Y^{2} \\
(X-I)^{2} Y & <(X-Y)^{2}
\end{aligned}
$$

The proof of the result then follows immediately.

\section{References}

[1] R.R. Bitmead, M.R. Gevers and I.R. Petersen, "Monotonicity and stabilizabiliy properties of solutions of the Riccati difference equation: propositions, lemmas, theorems, fallacious conjectures and counterexamples," Systems and Control Letters, Vol. 5, (1985), pp. 309-315.

[2] D. Cox, J. Little and D. O'Shara, "Ideals, varieties and algorithms: an introduction to computational algebraic geometry and commutative algebra." Undergraduate Texts in Mathematics, Springer Verlag, New York, 1992.

[3] C.E. García and M. Morari, "Internal model control 1: a unifying review and some new results," Ind. Eng. Chem. Proc. Des. Dev., Vol. 21, (1982), pp.308-323.

[4] K. Glover, "All optimal Hankel norm approximations of linear multivariable systems and their $L_{\infty}$ error bounds," Int. J. Cont., Vol.43, (1984), pp. 1115-1193.

[5] J. Ingham, I.J. Dunn, E. Heinzle, and J.E. Přenosil. "Chemical engineering dynamics: An introduction to modeling and computer simulation,” WILEY-VCH, Weinheim, 2000.

[6] W.H. Kwon and A.E. Pearson, "A modified quadratic cost problem and feedback stabilization of a linear system," IEEE Transactions on Automatic Control, Vol. 22, (1977), pp. 838-842.

[7] W.H. Kwon and K.B. Kim, "On stabilization receding horizon controls for linear continuous time-invariant systems," IEEE Transactions on Automatic Control, Vol. 45, (2000), pp. 13291334.

[8] J.H. Lee, "Recent advances in model predictive control and other related areas," Preprints of the Proceedings of the Chemical Process Control-V, Tahoe City 7-12 January, 1996.

[9] J.W. Lee, W.H. Kwon and J. Choi, "On stability of constrained receding horizon control with finite termal weighting matrix,” Automatica, Vol 34, (1998), pp. 1607-1612.

[10] K.R. Muske and J.B. Rawlings, "Model predictive control with linear models," AIChE Journal, Vol. 39, (1993), pp. 262-287.

[11] G. de Nicolao and S. Strada, "On the stability of receding horizon LQ cwith zero-state terminal constraint," IEEE Transactions on Automatic Control, Vol. 22, (1997), pp. 257-260.
[12] M.A. Poubelle, R.R. Bitmead and M.R Gevers, "Fake algebraic Riccati techniques and stability," IEEE Transactions on Automatic Control, Vol. 33, (1988), pp. 379-381.

[13] J.B. Rawlings and K.R. Muske, "The stability of constrained receding horizon control," IEEE Transactions on Automatic Control, Vol. 38, (1993), pp. 1512-1516.

[14] R. Scattolini and S. Bittanti, "On the choice of the horizon in long range predictive control -some simple criteria," Automatica, Vol. 26, (1990), pp. 915-917.

[15] A.A. Stoorvogel, S. Weiland and J. Ludlage, "On asymptotic stability of a receding horizon control scheme", Proceedings 38th IEEE Conference on Decision and Control, (1999).

[16] M. Sznaier and M.J. Damborg, "Heuristically enhanced feedback control of constrained discrete-time linear systems," Automatica, Vol. 6, (1990), pp. 521-532.

[17] M. Vidyasagar, "Control system synthesis: A factorization approach,” MIT press, Cambridge, MA, 1985.

[18] M. Vidyasagar, "Nonlinear system analysis," Prentice Hall, New Jersey, 1993. 